\section{PULMONARY FUNCTION AND NUTRITIONAL STATUS OF CHILDREN EXPOSED TO SMALL SCALE AGATE INDUSTRIAL UNITS IN SHAKARPUR, GUJARAT - INDIA}

doi:10.1136/archdischild-2012-302724.0674

${ }^{1,2}$ AS Nimbalkar, 'DV Patel, 'AR Sethi, ${ }^{3} \mathrm{~A}$ Patel, ${ }^{3} \mathrm{AG}$ Phatak, ${ }^{1,3} \mathrm{SM}$ Nimbalkar. 'Department of Pediatrics; ' $D$ Department of Physiology, Pramukhswami Medical College; ${ }^{3}$ Central Research Services, Charutar Arogya Mandal, Anand, India

Background In Shakarpur of Khambhat, a coastal city of Gujarat, India, several small agate polishing units operate from individual houses. Prevalence of Silicosis and other co-morbid conditions is systematically documented recently. Effect of environmental exposure on nutritional status and pulmonary function (PFTs) of children in this area was assessed.

Methods Cross sectional study was conducted in schools of this area. Weight was measured using standard digital bathroom scale while height was measured using Stadiometer (Seca). PFTs were measured for Forced Vital Capacity (FVC) and Forced Expiratory Volume in $1^{\text {st }}$ second (FEV1) using digital spirometer (One Flow FVC memo kit). Out of School children were not assessed.

Results 240 children (128 Boys and 112 Girls) in the age group of 10-16 years participated. 5 children ( 2 boys and 3 girls below 15 years of age) were working in agate industry. As per WHO growth standards $56.3 \%$ boys and $45.5 \%$ girls were stunted whereas $47.7 \%$ boys and $36.6 \%$ girls were undernourished. (Body Mass Index less than -2SD). The mean (SD) FVC [1.82(0.64) for boys vs. 1.83(0.63) for girls] and mean (SD) FEV1 [1.26(0.33) for boys vs. 1.29(0.34) for girls] was comparable across gender. No statistically significant difference was found in PFTs of children exposed to in house or neighboring agate industry as compared to unexposed children.

Conclusion PFTs are decreased in the entire population of children as compared to standards in Gujarat Population but agate exposed children did not show worse PFTs. Prevalence of under-nutrition in children was high.

\section{MALNOURISHED CHILDREN WITH ACUTE DIARRHEA}

doi:10.1136/archdischild-2012-302724.0675

TH Kamberi, M Azemi, M Avdiu, VI Jaha, VG Uka. Pediatrics, University Clinical Centre of Kosova, Prishtina, Kosovo

Introduction Diarrhoea continues to be a serious problem in children and may be fatal when superimposed upon malnutrition.

Objective To determine the frequency of electrolyte disturbances in malnourished children with diarrhea and whether these findings have therapeutic value or not

Methodology We included in the study 400 children age 6 months to 5 years admitted for acute watery diarrhea. On the basis of history, physical examination and anthropometrics measurement they were divided into Group A patients $(\mathrm{n}=116)$ who were malnourished and Group B patients $(\mathrm{n}=284)$ with normal nutrition. Serum electrolytes were done in patients of both groups and the results were analyzed statistically.

Results Analysis of serum electrolytes in both groups revealed that hypokalemia, hyponatremia and low serum bicarbonate were seen more frequently in patients of group $\mathrm{A}$ as compared to group B. In group A hypokalemia was seen in 39 patients $(39 / 116$ or $33.62 \%)$ while it was observed in 42 patients $(42 / 284$ or $14.78 \%)$ in group B $(p<0.001)$. Hyponatremia was seen in 21 patients $(21 / 116$ or $18.10 \%)$ in group A and in 14 patients (14/284 or $4.92 \%$ ) in group B $(\mathrm{p}<0.001)$. In group A 96 patients $(96 / 116$ or $82.7 \%)$ had low serum bicarbonate while in group B 122 patients (122/284 or $42.95 \%$ ) had low serum bicarbonate value $(\mathrm{p}<0.001)$.

Conclusion Electrolyte disturbances were commonly seen in malnourished children with acute diarrhea. The measurement of serum electrolytes is helpful for immediate therapy to avoid serious life threatening situation. Key words: Acute diarrhea, malnutrition, electrolytes.

\section{6 CONGENITAL CHLORIDE DIARRHEA: A REVIEW OF 12 ARAB CHILDREN}

doi:10.1136/archdischild-2012-302724.0676

F Alrefaee. Pediatrics, Adan Hospital, Kuwait, Kuwait

Background Congenital chloride diarrhea (CCD), a rare autosomal recessive disorder, is characterized by sustained watery diarrhea (due to defect of active Chloride/HCO3 exchange in the ileum and colon) with high fecal chloride. The estimated incidence of CCD was 1 in 14000 in Kuwait reported in 1989. Aim of our study was to spotlight the common presentation of infants with CCD which appears not to be uncommon disorder in Kuwait for early management and prevention of complications.

Subjects and methods Reviewing the inpatient database of Pediatric department of Al-Adan Hospital, Kuwait for patients who were admitted as chronic diarrhea in the first year of life, 12 patients diagnosed as CCD (7 females and 5 males). The diagnosis of CCD was based on antenatal and early infantile presentation and confirmed by laboratory finding of excess chloride loss in stool and low serum chloride level.

Results All 12 patients were born to consanguineous parents, had antenatal history of IUGR, polyhydramnios and distended hypoechoic fetal bowel and presented with abdominal distension, hypotonia and muscle wasting. Whereas, 10 patients (90\%) were delivered prematurely, 8 patients $(66.6 \%)$ had maternal hypertension, 9 patients $(75 \%)$ had absence of normal meconium at birth Our patients showed significant decrease in serum sodium, potassium, chloride \& urine chloride compared with the average for age. Conclusions High index of suspicious and awareness should be considered for early diagnosis of CCD in this population especially in the presence of consanguineous marriage.

\section{ELASTOGRAPHIC ASSESSMENT OF LIVER FIBROSIS IN CHILDREN WITH MALIGNANCIES VERSUS CHILDREN WITH HEPATIC STEATOSIS AND OTHER CHRONIC LIVER DISEASES}

doi:10.1136/archdischild-2012-302724.0677

O Marginean, C Marginean, K Brinzaniuc, A Pitea, S Voidazan. University of Medicine and Pharmacy Târgu-Mures, Târgu-Mures, Romania

The ultrasound elastography has more and more clinical applications, providing information related to the elasticity/stiffness of the examined-tissue. The aim of this paper was to assess the elastography performance for real-time ARFI in evaluating the liver fibrosis in children with malignancies, after-chemotherapy, compared with chronical liver diseases.

Material and Methods A prospective study was performed in Pediatric Clinic Ist Tg.-Mures, Romania, 2010, September 15th2011, September 15th, on 153 hospitalized children - 58 children with malignancies, 28 with liver diseases, 20 obese children and a control-group - 47 children. The liver tissue elasticity has been evaluated (Shear Wave Velocity, SWV) as well as some paraclinical parameters; statistical correlations were established.

Results and discussion Comparing the values of transaminasis, statistically significant differences were found between children with liver diseases and control-group regarding aspartate-aminotransferase, AST and alanine-aminotransferase, ALT.SWV was measured globally and separately for the liver-segments 1 and 8. In normal conditions in the $1^{\text {st }}$-segment SWV values were smaller than in $8^{\text {th }}$-segment $(p=0.02)$. In the group of obese children the SWV values were statistically significant bigger than in controls, the liver 
steatosis being correlated with bigger values of SWV. Positive statistical correlations have been established between AST and SWV in the group of obese chidren and after-chemotherapy.

Conclusions ARFI allows SWV quantification of the SWV in strong correlation with the fibrosis stage, the hepatic steatosis and the liver changes after chemotherapy.

"This paper is partially supported by the Sectoral Operational Programme Human Resources Development, financed from the European Social Fund and by the Romanian Government under the contract number POSDRU/89/1.5/S/60782".

\section{GASTROINTESTINAL TRANSIT PATTERNS IN CHILDHOOD CHRONIC CONSTIPATION}

doi:10.1136/archdischild-2012-302724.0678

1.2,3YI Yik, ${ }^{4} \mathrm{DJ}$ Cook, ${ }^{4} \mathrm{DM}$ Veysey, ${ }^{4} \mathrm{CF}$ Tudball, ${ }^{4} \mathrm{BS}$ King, ${ }^{4} \mathrm{KA}$ Morris, ${ }^{4} \mathrm{TM}$ Cain, ${ }^{3}$ BR Southwell, 2,3,5JM Hutson. 'Department of General Surgery, Faculty of Medicine, University of Malaya, Kuala Lumpur, Malaysia; '2Department of Paediatrics, University of Melbourne; ${ }^{3}$ Gut Motility and Research Laboratory, Murdoch Children's Research Institute; ${ }^{4}$ Department of Biomedical Imaging; ${ }^{5}$ Department of Urology, Royal Childrens Hospital Melbourne, Melbourne, VIC, Australia

Background and Aims Constipation may be a part of a generalized gastrointestinal (GI) tract disorder. Nuclear transit scintigraphy (NTS) provides transit through the stomach, small bowel, colon and anorectum. This study aimed to determine different colonic and rectal transit patterns in children with chronic constipation (CC) and their association with upper GI tract disorders.

Methods A retrospective analysis of NTS (1999-2011) performed in children with intractable CC. The 48-hour NTS protocol involved Gallium-67 citrate milk drink with images acquired at 0-2 hrs (gastric emptying study) and at 6, 24, 30 and 48 hrs (small bowel \& colonic transit studies). The geometric centre calculation was based on $\%$ of radioactivity in each region of interest (ROI). Six ROIs were employed ( $1=$ pre-colonic, $2=$ ascending colon, $3=$ transverse colon, $4=$ descending colon, $5=$ recto-sigmoid colon and $6=$ toilet).

Results A total of 955 NTS was performed (1999-2011; 288 repeat \& 667 new studies). In the 603 children (284 female, 2-23yrs, mean $8.5 \pm 4.1 \mathrm{yrs}$ ) included for this study, $19 \%$ had normal colonic transit (NT), $52 \%$ slow colonic transit (ST) and $29 \%$ rapid proximal colonic transit (RT, Table 1). Only $1 / 3$ of children had AR. About $20 \%$ of children had delayed gastric emptying \& delayed small bowel transit.

Conclusions There are 3 distinct colonic transit patterns in children with CC: normal, slow \& rapid. About $1 / 3$ of children with CC had AR at $48 \mathrm{hrs} \&$ was associated with NT, ST and RT. In addition, 21-24\% children with CC had upper GI tract transit disorders.

\section{INFLAMMATORY CHANGES AND CERAMIDE PROFILES IN RAT LIVER AFTER FETAL ASPHYCTIC PRECONDITIONING AND PERINATAL ASPHYXIA}

doi:10.1136/archdischild-2012-302724.0679
1,2E Vlassaks, ${ }^{2} \mathrm{M}$ Nikiforou, ${ }^{1,3} \mathrm{JSH}$ Vles, 1,2E Strackx, ${ }^{1} \mathrm{P}$ Martinez-Martinez, ${ }^{2} \mathrm{BW}$ Kramer, ${ }^{1,2}$ AWD Gavilanes. 'Mental Health and Neuroscience, Maastricht University; ${ }^{2}$ Pediatrics, ${ }^{3}$ Child Neurology, Maastricht University Medical Center, Maastricht, The Netherlands

Background and Aims Fetal (FA) and perinatal asphyxia (PA) are major causes of neonatal morbidity and death worldwide. Although most studies are focused on the brain, FA and PA are known to be associated with multi-organ disease. Therefore, as part of the systemic impact, we aimed to investigate the hepatic inflammatory response after asphyxia.

Methods A clinical relevant rat model was used, inducing global asphyctic insults to reflect the human pathophysiology. At different time points (acute and chronic) after FA and PA, we assessed hepatic inflammation, ceramide signaling and hepatocellular damage. Additionally, we assessed whether the combination of both insults (preconditioning) would have any protective effect on the liver.

Results FA induced significant changes in inflammatory cytokines and ceramide metabolism genes with increased interleukin (IL)- $1 \mathrm{~b}$ mRNA at $6 \mathrm{~h}$, increased mRNA levels of IL-6, LAG1 homolog ceramide synthase 1 and ceramide transporters at $24 \mathrm{~h}$ and finally, increased acid sphingomyelinase and sphingomyelin synthase 1 mRNA at 96h. Also PA induced an inflammatory response, with increased IL- 6 and IL-10 levels $2 \mathrm{~h}$ after birth. The combination of FA and PA (preconditioning) attenuated the inflammatory response, reflecting comparable IL-6 and IL-10 levels as control animals. 8 months after birth, no significant differences between groups were observed in hepatic mRNA levels for all cytokines and ceramide enzymes. Nevertheless, markers for hepatocellular damage, AST and ALP, showed increased levels when animals experienced FA and PA.

Conclusions FA and PA induce acute changes in hepatic cytokine and ceramide levels which may lead to hepatocellular damage in later life.

\section{INTRA-ABDOMINAL TUMORS IN CHILDREN}

doi:10.1136/archdischild-2012-302724.0680

E Blevrakis, T Tavladaki, AM Spanaki, E Vasilaki, S llia, E Geromarkaki, MD Fitrolaki, G Briassoulis. PICU, University Hospital of Heraklion, Heraklion, Greece

Aim The objective of the present study was to observe the histopathological pattern of intra-abdominal tumors in children less than 15 years.

Material and Methods The study was carried out at the Department of Pediatric Intensive Care Unit, Heraklion, during a period of 7 years, from March 2005 to March 2012. The histopathological and demographic data of 15 intra-abdominal tumors of both sexes ( 8 boys and 7 girls) under 16 years of age was collected and analyzes to determine the various morphological types of intra-abdominal tumors in relation to age and sex.

Results Neuroblastoma was the most common tumor constituting $46.7 \%$ of all cases, followed by Wilms'tumor (26.7\%), hepatoblastoma (13.2), teratoma and granulosa cell tumor (6.7\%) each. Majority of the patients $73.3 \%$ were under 5 years of age.

Abstract 678 Table 1

\begin{tabular}{llcccccc}
\hline Proximal colonic transit & \multicolumn{2}{c}{ Gastric emptying } & Small bowel transit & $\begin{array}{c}\text { Distal colonic } \\
\text { transit }\end{array}$ & Anorectal transit \\
\hline & Normal & \multicolumn{1}{c}{ Delayed } & Normal & Delayed & Slow & Normal & retention (AR) \\
\hline Normal (NT) & $91(15 \%)$ & $23(4 \%)$ & $82(14 \%)$ & $32(5 \%)$ & - & $62(10 \%)$ & $52(9 \%)$ \\
Slow (ST) & $251(42 \%)$ & $63(10 \%)$ & $221(36 \%)$ & $93(16 \%)$ & - & $237(39 \%)$ & $77(13 \%)$ \\
Rapid (RT) & $135(22 \%)$ & $40(7 \%)$ & $156(26 \%)$ & $19(3 \%)$ & $21(4 \%)$ & $74(12 \%)$ & $80(13 \%)$ \\
TOTAL & $477(79 \%)$ & $126(21 \%)$ & $459(76 \%)$ & $144(24 \%)$ & $21(4 \%)$ & $373(61 \%)$ & $209(35 \%)$ \\
\hline
\end{tabular}

